\title{
Mindfulness and the Social Media
}

\section{Edo Shonin*, William Van Gordon and Mark D Griffiths}

Psychology Division, Nottingham Trent University, Nottinghamshire, UK

*Corresponding author: Division of Psychology, Chaucer Building, Nottingham Trent University, Burton Street, Nottingham, UK, Tel: +44 (0)115 8484460; E-mail: e.shonin@awaketowisdom.co.uk

Rec date: Mar 11, 2014; Acc date: May 28, 2014; Pub date: June 04, 2014

Copyright: (c) 2014 Shonin E, et al. This is an open-access article distributed under the terms of the Creative Commons Attribution License, which permits unrestricted use, distribution, and reproduction in any medium, provided the original author and source are credited.

\begin{abstract}
The number of people engaging in and using social media has increased substantially over the past decade. Recent research has also suggested that a minority of individuals appear to be addicted to social media (e.g., socalled 'Facebook addiction'). Another area that has seen substantial growth during the same time period is the use of mindfulness. Following a brief overview of both excessive social media use and the basic concepts relating to what mindfulness is, this paper briefly reviews some of the main issues in these two areas and suggests that mindfulness might be a useful therapeutic intervention that alleviates problems associated with social media use (given that mindfulness has been used successfully in the treatment of other behavioral addictions such as addictions to gambling and work). In light of the few studies carried out on excessive social media usage, it is concluded there is a need for more methodologically robust research exploring the prevalence, aetiology, and correlates of maladaptive social media behaviour.
\end{abstract}

Keywords: Social media usage; Social media addiction; Facebook addiction; Mindfulness; Meditation awareness training

\section{Introduction}

Over the last decade, there has been a significant increase in the number of people engaging in and using social media. For instance, almost three-quarters of adult internet users (73\%) now use at least one social media site and $42 \%$ use multiple social media sites (e.g., Facebook, MySpace, LinkedIn, Pinterest, Twitter, Instagram, etc.) [1]. Additionally, approximately $50 \%$ of adolescents use social media sites more than once per day, and $22 \% \log$ on to social media sites more than ten times per day [2]. Research demonstrates that social media engagement can be life enriching and enhance communication skills, creativity, social connectedness (including in offline social networks), knowledge acquisition, and technical proficiency [3]. However, despite these possible benefits, excessive social media usage in a minority of individuals can also incur serious negative psychosocial consequences including (but not limited to) (i) addiction (e.g., to a specific social media site, to social networking more generally, to the internet, and/or to a fixed or portable internet device), (ii) relationship breakdown, (iii) academic drop-out and underachievement, (iv) escapism and avoidance, (v) cyber-bullying and cyber-harassment, (vi) dissemination of untrustworthy information, (vii) sleep deprivation, and (viii) psychopathology $[2,4,5]$.

Known factors that affect how social media influences an individual's wellbeing and behaviour include (for example) frequency and duration of social media usage, motivation underlying use, age, personality type, psychiatric history, and the structural characteristics of the social media software [4-7]. However, consistent with the findings of research investigating the role of metacognitive awareness on task engagement and psychosocial functioning, we assert that the attentional strategy employed when using social media is a key determinant of whether such usage becomes life enriching or life impoverishing. Of particular interest in this regard is the attention- based phenomenon known as mindfulness. Scientific interest concerning mindfulness has increased substantially in recent years particularly in terms of its utility for fostering psychological wellbeing amid the pressures and demands of contemporary living. Following a brief introduction to mindfulness, we briefly assess the potential applications of mindfulness in the ongoing assimilation of social media by users from increasingly diverse sectors of society.

\section{What is Mindfulness?}

Mindfulness derives from Buddhism and is a form of meditation. It is defined as "the process of engaging a full, direct, and active awareness of experienced phenomena that is spiritual in aspect and that is maintained from one moment to the next" [8]. The traditional Buddhist teachings on mindfulness assert that in general, people are rarely aware of the present moment and that the majority-share of their cognitive resources are essentially wasted due to dwelling in and being preoccupied with the future, or being unable to let go of the past [9]. Indeed, the traditional literature on mindfulness asserts that with the exception of accomplished meditators (or spiritual practitioners), the average person is afflicted by 'mindlessness' and is plugged-in to a reality other than the here and now [9].

Mindlessness refers to the condition whereby a person goes through their life without ever truly being present with themselves and without being adequately awake to fully experience and embrace each instant of the present moment. Given the present moment is the only place where life can actually be experienced (because the future is "fantasy" and the past is "history") [9], Buddhist teachings assert that the regular practice of mindlessness results in stress, exhaustion, mental illness, and spiritual undernourishment [9]. We have previously defined mindlessness as a form of 'inverted hallucination' due to it involving 'the non-perceiving of that which is' [9]. This is a slight re-ordering of the conventional understanding of hallucination that can be defined as the 'perceiving of that which is not'. Thus mindfulness - the opposite of mindlessness - is fundamentally concerned with becoming more 
aware of the present moment and with recovering from a chronic inverted-hallucination in order to embrace and reconnect with the here and now.

\section{Mindfulness for Adaptive Social Media Engagement}

As already indicated above, there are numerous potential adverse effects that have been associated with excessive social media usage. However, the most commonly reported negative consequences arguably include (i) excessive/problematic use and addiction, (ii) antisocial and/or illegal behaviour (e.g., cyber-bullying, cyber-harassment, sexting, etc.), and (iii) psychopathology $[2,4,5]$. We now briefly address each of these individual areas of concern and provide an evidence-based discussion of how mindfulness may be able to regulate potentially maladaptive social media usage.

\section{Addiction}

Although Internet Use Disorder was not included in the main text of the American Psychiatric Association's Diagnostic and Statistical Manual of Mental Illness (i.e., the DSM-5), it was included in Section 3 ['Emerging Measures and Models'] indicating that online addictions (such as social media addiction, online gaming addiction, etc.) may be a valid construct for some excessive users. For example, prevalence estimates of internet addiction in adolescents vary considerably but are typically in the order of 5-18\% [10]. Of course, social media addiction is only one possible variant of addictions on and/or to the internet (i.e., in addition to online gambling addiction, online shopping addiction, computer game addiction, web surfing addiction, cybersexual addiction, etc.) and there are few reliable estimates of the proportion of individuals exhibiting internet addiction symptomatology that are exclusively addicted to social media [4]. Nevertheless, emerging evidence indicates that social media addiction can incur serious negative health consequences among a minority of users and is likely to increase in prevalence as increasingly more individuals frequent social media sites [2,4-7].

Although there are few (if any) empirical studies specifically investigating the role of mindfulness in the prevention and treatment of social media addiction, it is plausible to assume that mindfulness techniques with demonstrable efficacy in the treatment of other forms of internet addiction (i.e., online gambling addiction) are likely to have utility in reducing problematic or pathological social media engagement. Indeed, based on the components model of addiction [11] the following addiction criteria are believed to occur in all forms of behavioural addiction (including social media addiction) irrespective of addiction type: (i) salience (i.e., cognitive, behavioural, and emotional preoccupation with social media usage), (ii) mood modification (i.e., social media engagement leads to a favourable change in emotional states), (iii) tolerance (i.e., ever increasing usage of social media over time), (iv) withdrawal (i.e., experiencing unpleasant physical and emotional symptoms when social media use is restricted or stopped), (v) conflict (i.e., psychological and interpersonal problems ensue because of social media usage), and (vi) relapse (i.e., individuals quickly revert back to excessive social media engagement following a period of abstinence) [5].

Despite these underlying similarities between different classes of behavioural addiction, an important clinical implication concerning social media addiction is that unlike most other forms of addiction, treatment outcomes for social media addiction cannot realistically be focused on total abstinence but must be orientated towards control (as trying not to access the internet in contemporary society is almost impossible)[4]. Recently, in addition to specifically highlighting the likely utility of mindfulness as a control-based treatment for social media addiction [8], we conducted a review and assessment of the particular mechanisms by which mindfulness targets each of the above outlined behavioural addiction components [12]. Of particular mechanistic importance in this respect, is the increase in perceptual distance from sensory and cognitive-affective stimuli that is a commonly-experienced outcome of mindfulness practice [13]. By objectifying their cognitive and affective processes in this manner, individuals addicted to social media are better able to regulate habitual compulsive responses and adopt an observatory, non-judgemental, and non-reactive attentional-set towards mental urges (known as urge surfing) [8]. Consequently, mindfulness facilitates reductions in impulsivity and salience due to individuals understanding that their cravings are transient phenomena and are without inherent worth [8]. Given the difficulties that online users face in creating distance from social media (e.g., due to constant audible activity alerts and notifications via their mobile phone, tablet, laptop, and/or personal computer), this ability to simply observe and not immediately react to both sensory (e.g., audible notification) and psychological (e.g., mental urges) stimuli is likely to be a particularly pertinent outcome of mindfulness in terms of its application as a social media addiction treatment.

Other important mechanisms of action by which mindfulness may modulate the symptoms of social media addiction include: (i) reductions in relapse and withdrawal symptoms via substituting addiction to social media with a 'positive addiction' to mindfulness/ meditation (particularly the tranquil states associated with certain concentrative meditation practices) [8], (ii) reductions in salience and sensation-seeking by undermining the authenticity that individuals assign to their involvement with social media (i.e., due to a better understanding of the impermanent nature of phenomena and a reevaluation of life priorities) $[8,12]$, and (iii) increased patience that leads to an improved capacity to defer gratitude and a reduced need to be constantly plugged-in [8]. Mindfulness might be a useful therapeutic intervention that alleviates problems associated with social media use given that mindfulness has been used successfully in the treatment of other behavioral addictions such as addictions to gambling [14] and work [15].

\section{Antisocial and Illegal Behaviour}

There appears to be consensus in the scientific literature that online antisocial and illegal behaviour (e.g., cyber-bullying, cyberharassment, privacy issues, sexting, etc.) are a means by which certain users abuse social media sites to the detriment of all. Adolescent user groups are frequently implicated in this respect [2], although such issues and behaviour are certainly not limited to non-adult populations.

Qualitative findings investigating participant experiences of receiving an eight-week secular mindfulness intervention known as Meditation Awareness Training (MAT) suggest that mindfulness (and related meditative techniques) leads to a greater awareness not only of the emotions and suffering of the participant, but also the emotions and suffering of others [16]. Accordingly, mindfulness is associated with increases in self-compassion and compassion as well as improvements in citizenship, pro-social behaviour, and generosity [9]. Furthermore, concepts such as interconnectedness are often integrated into the curriculum for mindfulness interventions and there is 
evidence suggesting that awareness of the consequences of thoughts, words, and actions increases in line with mindfulness competency [9]. Thus, mindfulness, which has been shown to be an acceptable intervention for multiple age groups (including adolescents), may encourage "offending" individuals to refrain from antisocial and illegal behaviour on social media sites and take greater responsibility for the content of their online output. A recent Mindfulness in Practice article published in the academic journal Mindfulness explains this principle further:

- Each of our thoughts, words, and actions dictate who we are now and who we will be in the future. Those same thoughts, words, and deeds also influence who others will be in the future. Therefore, the next time you write something or create a product for other people's minds, perhaps you might like to consider how your "mental food" will affect the wellbeing of the consumers. It should be reasonably easy to tell where somebody is writing with awareness because their words should be easily absorbed and should be alive with wisdom. Such words should effortlessly fly off the page and speak directly to your heart. Reading mindful words should leave us feeling spiritually nourished, calmer, and with a clearer perspective. Mindful words should help us to stop and be, to let go a little, and to feel bathed and refreshed by that person's compassion and awareness. Mindful words should help us to remember that we came into this world all alone, and that at some uncertain point, we will certainly have to leave this world all alone. ... Perhaps we could say that words written with mindfulness provide us with all five of our "spiritual five a day" [15].

Obviously, mindfulness should not be seen as a fix-all solution to antisocial behaviour on social media sites because the effective development of mindfulness requires a significant commitment on behalf of the participant (and it is probably safe to assume that there are considerable number of users that would not consider mindfulness to be an attractive option) [17]. However, findings from a recent nationally-representative survey of British adults demonstrate that mindfulness is growing in popularity amongst the general public and that $86 \%$ of people believe that being mindful of the present moment can improve their health and happiness [18]. Thus, it is not unreasonable to assert that there may be an important role for mindfulness as part of initiatives seeking to operationalise social media sites as portals for life-enrichment and more responsible cyberinteraction.

\section{Psychopathology}

Whether in relation to addiction or antisocial behaviour, or as isolated consequences, psychopathology and psychological issues are associated with problematic social media engagement. More specifically, research has correlated excessive social media usage with (i) low self-esteem (e.g., due to receiving negative feedback from peers and/or anonymous third parties), (ii) asociality (e.g., increased physical isolation from family and peers), (iii) dysfunctional coping (e.g., escapism and avoidance), and (iv) dysphoric mood states (e.g., depression including so-called "Facebook depression") $[2,4,7]$.

There already exists a credible body of evidence indicating that mindfulness is effective for treating mood disorders and negative affective states. Indeed, mindfulness is advocated by both the UK National Institute for Health and Care Excellence and the American Psychiatric Association for the treatment of recurrent depression in adults. Furthermore, meta-analytical effect sizes for mindfulness in the treatment of mood and anxiety disorders are typically large in magnitude (i.e., $\mathrm{d}>0.8$ ), with therapeutic gains typically being maintained at standard-term follow-up assessment points (i.e., three or six months post-intervention).

Key mechanisms of action underlying the salutary treatment effects of mindfulness on mood disorders (and on psychopathology more generally) include (i) a growth in spiritual awareness that broadens perspective and buffers against loneliness and low self-esteem [9], (ii) transference of the locus of control for stress from external conditions to internal metacognitive and attentional resources [19], (iii) the modulation of shameful and self-disparaging schemas via the cultivation of self-compassion [8], (iv) reduced autonomic and psychological arousal via conscious-breathing [20], and (v) a greater ability to label and therefore modulate faulty thinking patterns $[21,22]$.

\section{Conclusions}

Recent years have witnessed a significant increase in the use of social media and many individuals derive both short- and long-term benefits from their social networking activity. However, the increasing ease at which technology allows (or encourages) access to social media means that a small minority of individuals appear to experience difficulty in moderating their online usage. Accordingly, excessive social media engagement is associated with a variety of maladaptive psychosocial outcomes, and there is a need for novel strategies that can ameliorate such deleterious effects. Emerging clinical evidence suggests that mindfulness may be an effective response in this respect, and it appears that public interest and awareness of mindfulness are also increasing. Given the few studies carried out on excessive social media usage, there is a need for more methodologically robust research exploring the prevalence, aetiology, and correlates of maladaptive social media behaviour more generally.

\section{References}

1. Duggan M, Smith A (2014) Social media update 2013. Pew research centre.

2. O'Keeffe GS, Clarke-Pearson K (2011) The impact of social media on children, adolescents, and families. Paediatrics 127: 800-804.

3. Ito M, Horst H, Bittani M, Boyd D, Herr-Stephenson B, et al. (2008) Living and learning with new media: Summary of findings from the digital youth project.

4. Kuss DJ, Griffiths MD (2011) Online social networking and addiction: A literature review of empirical research. International Journal of Environmental and Public Health 8: 3528-3552.

5. Griffiths MD (2013) Social networking addiction: Emerging themes and issues. Journal of Addiction Research and Therapy 4: e118.

6. Griffiths MD (2000) Does internet and computer "addiction" exist? Some case study evidence. Cyberpsychology and Behavior 3: 211-218.

7. Griffiths MD, Kuss DJ, Demetrovics Z (2014) Social networking addiction: An overview of preliminary findings.

8. Shonin E, Van Gordon W, Griffiths MD (2014) Mindfulness as a treatment for behavioral addiction. Journal of Addiction Research and Therapy 5: e122.

9. Shonin E, Van Gordon W, Griffiths MD (2014) The emerging role of Buddhism in clinical psychology: Toward effective integration. Psychology of Religion and Spirituality 6: 123-137.

10. Wallace P (2014) Internet addiction disorder and youth. EMBO Reports 15: 12-16.

11. Griffiths MD (2005) A 'components' model of addiction within a biopsychosocial framework. Journal of Substance Use 10: 191-197. 
Citation: Shonin E, Van Gordon W, Griffiths MD (2014) Mindfulness and the Social Media. J Mass Communicat Journalism 4: 194. doi: 10.4172/2165-7912.1000194

Page 4 of 4

12. Shonin E, Van Gordon W, Griffiths MD (2013). Buddhist philosophy for the treatment of problem gambling. Journal of Behavioural Addictions 2 63-71.

13. Van Gordon W, Shonin E, Zangeneh M, Griffiths MD (2014) Workrelated mental health and job performance: Can mindfulness help? International Journal of Mental Health and Addiction 12: 129-137.

14. Shonin E, Van Gordon W, Griffiths MD (2014) Cognitive Behavioral Therapy (CBT) and Meditation Awareness Training (MAT) for the treatment of co-occurring schizophrenia with pathological gambling: A case study. International Journal of Mental Health and Addiction 12 181-196.

15. Shonin E, Van Gordon W, Griffiths MD (2014). The Treatment of Workaholism with Meditation Awareness Training: A case study. Explore: Journal of Science and Healing 10: 193-195.

16. Shonin E, Van Gordon W, Griffiths MD (2014) Meditation Awareness Training (MAT) for improved psychological wellbeing: A qualitative examination of participant experiences. Journal of Religion and Health 53: 849-863.
17. Shonin E, Van Gordon W (2014) The consuming mind. Mindfulness 5: 345-347.

18. Mental Health Foundation (2010) Mindfulness Report. London.

19. Van Gordon W, Shonin E, Sumich A, Sundin E, Griffiths MD (2013) Meditation Awareness Training (MAT) for psychological wellbeing in a sub-clinical sample of university students: A controlled pilot study. Mindfulness, DOI: 10.1007/s12671-012-0191-5.

20. ShoninE,Van Gordon W, Griffiths MD (2013) Do mindfulness-based therapies have a role in the treatment of psychosis? Australia and New Zealand Journal of Psychiatry 48: 124-127.

21. Shonin E, Van Gordon W, Griffiths MD (2013) Mindfulness-based interventions: Towards mindful clinical integration. Frontiers in Psychology 4: 1-4.

22. Shonin E, Van Gordon W, Slade K, Griffiths MD (2013) Mindfulness and other Buddhist-derived interventions in correctional settings: A systematic review. Aggression and Violent Behavior 18: 365-372. 